\title{
CARGA DE TRABALHO E DIMENSIONAMENTO DE PESSOAL DE ENFERMAGEM EM UNIDADES DE TERAPIA INTENSIVA
}

\author{
WORKLOAD AND NURSING STAFF SIZING IN INTENSIVE CARE UNITS

\section{Clesnan Mendes-Rodrigues ${ }^{a}$, Karine Ellen Soares Costa ${ }^{b}$, Arthur Velloso Antunes ${ }^{c}$, Fabíola Alves Gomes ${ }^{d}$, Geraldo Júnior Rezendee, Durval Veloso Silva ${ }^{f}$} \\ aclesnan@hotmail.com, bkarine.ellencosta@gmail.com, carthurantunes22@gmail.com, dfabiola-gomes@uol.com.br, \\ eg.jr.rezende@bol.com.br, ${ }^{\mathrm{f}}$ durvals@hc.ufu.br \\ Universidade Federal de Uberlândia - Uberlândia (MG), Brasil \\ Data de recebimento de artigo: 27/06/2016 \\ Data de aceite do artigo: 02/06/2017
}

\section{RESUMO}

Embora escores com intuito de calcular a carga de trabalho em enfermagem sejam comuns, ainda são pouco utilizados na realidade brasileira de Unidades de Terapia Intensiva (UTI). Nesse contexto, o objetivo do trabalho foi avaliar o Nursing Activities Score (NAS) em quatro UTI de um Hospital Universitário, e verificar a relação do NAS com o Sistema de Classificação de Pacientes (SCP), proposta por Santos et al., em uma UTI Adulto, e o efeito sobre o dimensionamento de enfermagem. Todos os escores tiveram distribuição assimétrica e mostraram-se concentrados nas primeiras classes de distribuição. O NAS foi dependente do plantáo de avaliaçấo somente para as duas unidades de UTI Neonatal, enquanto não diferiu entre as demais. As UTI Neonatal Tipo III e a Pediátrica indicaram os maiores escores médios e a Neonatal Tipo II o menor. Apesar das diferenças entre plantóes para os escores do NAS, o dimensionamento não foi dependente do plantáo. O SCPA e o NAS levaram a um dimensionamento diferente, sendo maior para o último, mesmo os dois mostrando-se correlacionáveis. O NAS apresentou boa aplicabilidade nas unidades avaliadas e o sistema de classificaçáo de paciente mostrou-se pouco efetivo em discriminar os pacientes na UTI Adulto, principalmente pela baixa representatividade de pacientes de cuidados mínimos e intermediários. A presença de diferenças entre plantôes pode estar relacionada ao maior conhecimento dos pacientes da equipe da manhã, reforçando a necessidade de compreender a avaliação de paciente por diferentes profissionais ou momentos.

Palavras-chave: Dimensionamento; enfermagem; sistema de classificação de pacientes; carga de trabalho; nursing activities score.

\section{ABSTRACT}

Although scores to calculate the workload in Nursing are common, they are not used regularly in Brazilian intensive care units (ICU) routine. In this context, the objective of this work was to evaluate the Nursing Activities Score (NAS) in four University Hospital's ICU, and verify the relation between System of Classification of Patients (SCP), proposed by Santos at al., in an Adult ICU and the effect on nursing sizing. The work was developed in a university hospital. All scores had asymmetric distribution and were concentrated in the first classes of distribution. The NAS was dependent on the shift of evaluating only for the two ICU Neonatal units, while there was no difference between the others. The Pediatric and Neonatal type III ICU had higher average scores and the Neonatal type II show the lower. Despite the differences between shifts to the scores of the NAS, the sizing was not dependent on the shift. The ASCP and the NAS led to a different scaling, the latter being higher, although they are correlated. The NAS has shown good applications to evaluated units and the patient classification system proved little effective in discriminating Adult ICU patients, mostly by low representation of minimum and intermediate care patients. The presence of differences between shifts may be related to greater knowledge of the patients by the morning team, reinforcing the need to understand the patient classification by different professionals or moments.

Keywords: Sizing; nursing; patient classification system; workload; nursing activities score. 


\section{Introdução}

A definição da carga de trabalho em enfermagem e o dimensionamento de pessoal de enfermagem são temáticas que permeiam o dia a dia dos enfermeiros, desde a atividade diária de elaboração das escalas de atribuição até a organização e dimensionamento de pessoal da instituição, sendo parte obrigatória das funções do enfermeiro. Para esse propósito, são utilizados instrumentos para aferição dessa carga de trabalho existente, que utilizam o perfil de cuidado para definição do tempo de assistência de enfermagem ${ }^{1,2}$, evitando serem influenciados pelas experiências subjetivas e individuais do enfermeiro.

Para as unidades de terapia intensiva o instrumento de mensuração da carga de trabalho mais utilizado é o Nursing Activies Score (NAS), um instrumento de avaliação retrospectiva ${ }^{1}$, em que a pontuação 100 equivale a 24 horas de assistência de enfermagem. No Brasil, esse instrumento foi validado por Queijo e Padilha ${ }^{3}$ e tem sido utilizado por muitos serviços. Alguns estudos têm demonstrado o efeito do horário de avaliaçáo (turno de trabalho) no resultado do NAS médio das unidades ${ }^{4} \mathrm{e}$ as diferenças entre subunidades de um mesmo serviço ${ }^{5}$, fatores esses que podem ter impactos diretos sobre o dimensionamento de pessoal desses serviços.

Outro instrumento de classificação amplamente utilizado no Brasil é o Sistema de Classificação de Pacientes (SCP), de Santos et al. ${ }^{2}$, proposto para avaliação de pacientes adultos, que classifica o paciente em função da demanda de cuidado no momento da avaliação. É adotado pelos Conselhos de Enfermagem para realização do dimensionamento de enfermagem e usa uma escala de pontuaçáo que classifica os pacientes em quatro tipos de cuidado, cada um com uma carga de trabalho ${ }^{2}$.

Tanto o NAS como o SCP servem para mensurar a carga de trabalho de enfermagem, sendo utilizados em Unidades de Terapia Intensiva (UTI) e em setores de internação de adultos, respectivamente. A relação entre os resultados obtidos por esses dois instrumentos na mensuração da carga de trabalho ainda não foi completamente estudada e, portanto, não é totalmente conhecida. Nesse sentido, o único exemplo que obtivemos foi um estudo em que o NAS foi utilizado para a determinação da carga de trabalho de enfermagem para pacientes de alta dependência ${ }^{6}$. Estudos relacionais têm focado mais na relação do NAS com os preditores de morbimortalidade e/ou com os desfechos clínicos $^{7}$. Ainda são incipientes a utilizaçáo e a criação de SCP para pacientes pediátricos ou neonatológicos ${ }^{8,9}$ e até mesmo a utilização de outros sistemas como o NAS nessas respectivas áreas ${ }^{10}$. Embora esses sistemas tenham sido validados, há ainda poucos exemplos de seu uso na literatura; o que não permite uma comparação direta com os resultados obtidos pela utilização do NAS e dos SCP.

\section{Objetivo}

Nesse contexto os objetivos do trabalho foram caracterizar e verificar a diferença do dimensionamento de pessoal de enfermagem a partir de três turnos de classificação distintos utilizando o NAS em unidades de Terapia Intensiva Adulto, Pediátrica e Neonatal em um hospital universitário; além de testar se em UTI Adulto o uso do sistema de classificação de pacientes de Santos et al. ${ }^{2}$ produz resultados diferentes de dimensionamento de pessoal daqueles obtidos pelos NAS, e a relação entre os dois instrumentos.

\section{Material e métodos}

\section{Área de estudo e aspectos legais}

Os dados utilizados para a caracterização das UTI no estudo foram obtidos a partir de dados coletados para o dimensionamento de pessoal em enfermagem do Hospital de Clínicas da Universidade Federal de Uberlândia (HC-UFU), Minas Gerais, Brasil ${ }^{11}$. O trabalho não fere nenhum aspecto ético e legal, visto que nenhum paciente pode ser identificado.

As avaliaçóes foram executadas de março a maio de 2013 e foram realizadas em todas as UTI do HC-UFU. A classificação de carga de trabalho de enfermagem em UTI faz parte das exigências legais dos serviços de saúde (Art. 4 Inc. XXIII, RDC no 7) ${ }^{12}$, e não ferem nenhum princípio ético e/ou legal. Por essa razão, e por não fazer parte das exigências legais, o perfil sociodemográfico e clínico dos pacientes não foi estabelecido.

A UTI Adulto conta com 30 leitos que atendem a todos os perfis de pacientes, adolescentes e/ou adultos (a partir de 13 anos); a UTI Pediátrica conta com 8 leitos e atende a crianças de 29 dias a 12 anos e 11 meses; e a UTI Neonatal atende a pacientes de até 28 dias e é dividida - em função da classificação estabelecida na Portaria GM/MS no $3.432 / 98^{13}$ - em UTI Neonatal II (que dispóe de estrutura para atendimento a casos de menor complexidade), com 5 leitos e em UTI Neonatal III (que dispõe de estrutura para atendimento a casos de maior complexidade), com 10 leitos. 
O trabalho foi submetido e aprovado pelo Comitê de Ética em Pesquisa da Universidade Federal de Uberlândia, sob o parecer de número 649.859/2013, e segue as recomendações da Resolução do Conselho Nacional de Saúde 466/2012.

\section{Nursing Activities Score nas diferentes UTI}

Foram realizadas 8 avaliações em cada unidade de terapia intensiva do HC-UFU, sendo que para cada uma foram avaliados todos os pacientes internados e presentes na unidade naquele momento. Sempre a avaliação foi executada pelo enfermeiro assistencial escalado na unidade de internação e por um enfermeiro "auditor" externo à Unidade. Nessas avaliaçóes foi adotado o Nursing Activities Score, NAS ${ }^{1}$, validado para o Brasil ${ }^{3}$.

Foram adotados três turnos de classificação, aqui denominados turnos da manhã (entre 06:30 e 12:30), tarde (entre 12:30 e 18:30) e noite (entre 18:30 e 00:30). Todas as unidades foram classificadas 3 vezes no período da manhã e da tarde, e 2 vezes no período da noite. $\mathrm{O}$ horário de cada turno da classificação e as datas entre elas não foram padronizados.

\section{Relação entre NAS e SCP adulto}

Para avaliar a relação entre o NAS e o SCP foram realizadas avaliaçóes pareadas para os pacientes da UTI Adulto em seis datas distintas. Estas datas não coincidem com a análise anterior e os dados não entraram na comparação entre as UTI. Para cada paciente foi realizada a classificação em função do NAS e do SCP. Todos os pacientes, ou seus representantes legais, que aceitaram participar da pesquisa assinaram o Termo de Consentimento Livre Esclarecido e somente foram incluídos pacientes maiores de 18 anos. Para esses pacientes foi adotado o SCP Adulto adaptado de Santos et al. ${ }^{2}$, com ajuste dos intervalos de 12 a 20 pontos para a classificação de cuidados mínimos; de 21 a 30 pontos para intermediários; de 31 a 39 pontos para semi-intensivos; e de 40 a 48 pontos para intensivos. Foram adotadas as cargas de trabalho de 3,8; 5,6; 9,4 e 17,9 horas/dia respectivamente, para cada tipo de cuidado ${ }^{14}$.

\section{Dimensionamento de pessoal de enfermagem}

\section{Sistema de classificação de pacientes}

Foram definidas as fórmulas a serem utilizadas para os cálculos dos profissionais de enfermagem dos setores de internação, optando-se pela mesma empregada no dimensionamento anteriormente feito pela Diretoria de Enfermagem, proposta por Antunes e Costa ${ }^{15}$, que é equivalente àquela proposta por Gaidzinski ${ }^{16}$ e pela Resolução Cofen no 293/04 ${ }^{14}$. Tal fórmula considera: o tipo de cuidado requerido pelos pacientes internados, que é determinado pela utilização do Sistema de Classificação de Pacientes (SCP); as horas de enfermagem requeridas para cada tipo de cuidado e o Índice de Segurança Técnica (IST). O dimensionamento seguiu a legislação vigente no ano de coleta dos dados, 2013.

Foram adotadas a lotação máxima de 30 leitos; a frequência de pacientes de cada um dos tipos de cuidados, obtida a partir da frequência relativa; a respectiva carga de trabalho, como preconizadas pelo Cofen ${ }^{14}$; o funcionamento durante os 7 dias da semana; a carga horária semanal de trabalho de 36 horas; e o índice de segurança técnica de $30 \%$.

\section{Nursing Activities Score}

O dimensionamento da equipe de enfermagem da UTI pode ser expresso pela fórmula $\mathrm{PE}=$ (E.L.MNAS/100).(1+IST/100), em que: PE número de profissionais de enfermagem necessários; $\mathrm{E}=$ número de turnos de enfermagem; $\mathrm{L}$ = número de leitos; MNAS = média de pontos do NAS; IST = Índice de Segurança Técnica. O dimensionamento foi realizado para cada dia de avaliação.

\section{Análise estatística}

Todos os dados foram testados previamente para normalidade com o teste de Kolmogorov-Smirnov/ Lilliefors. Para a comparação do NAS médio entre os turnos de cada UTI, e entre elas; e do dimensionamento com base no NAS diário, como os pressupostos da ANOVA não foram aceitos (normalidade e homocedasticidade), a comparação foi realizada pelos testes de Kruskal-Wallis e de Dunn para comparaçóes múltiplas. Para comparação da distribuição da carga de trabalho, foram construídos histogramas de frequência para cada uma das unidades avaliadas.

Para comparaçáo dos dados de dimensionamento NAS e SCP pelos dois métodos, que apresentaram normalidade, as médias foram comparadas entre si com o teste $t$ de Student para dados pareados. Os dados foram correlacionados entre si com uso da correlaçáo de Spearman (ausência de normalidade bivariada testada com o teste de Shapiro-Wilk para dados multivariados). Para o número de horas de enfermagem por paciente, 
obtidos pelo SCP e pelo NAS, que não apresentaram normalidade, as médias foram comparadas com o uso do teste de Mann-Whitney. A independência do tipo de cuidado com o dia de avaliação foi testada por meio do teste G com correção de Williams. Os dados dos escores pareados do SCP e do NAS foram ajustados a um modelo de regressão linear, que foi testado com uso de ANOVA, sendo testados os modelos com e sem intercepto.

Todas as análises estatísticas utilizaram uma significância de 5\%. Os testes foram realizados no Programa BioEstat $5.3^{17}$ ou no ambiente R (Versão 3.2.3).

\section{Resultados}

\section{Nursing Activities Score nas diferentes UTI}

A UTI Pediátrica mostrou distribuição normal dos dados de NAS, sendo que os dados das demais UTI mostraram distribuição náo normal, evidenciando um perfil bem característico para pacientes em algumas faixas de horas de enfermagem (Tabela 1).

Tabela 1: Estatísticas descritivas para os resultados da classificação da carga de trabalho em Enfermagem em Unidades de Terapia Intensiva pelo Nursing Activities Score (NAS) e pelo Sistema de Classificação de Pacientes (SCP) em um hospital universitário brasileiro.

\begin{tabular}{|c|c|c|c|c|c|c|}
\hline \multirow[b]{2}{*}{ Estatística } & \multicolumn{4}{|c|}{ NAS para as UTI } & \multicolumn{2}{|c|}{ UTI de Adultos } \\
\hline & Adulto & $\begin{array}{c}\text { Neonatal Tipo } \\
\text { II }\end{array}$ & $\begin{array}{c}\text { Neonatal Tipo } \\
\text { III }\end{array}$ & Pediátrica & NAS & SCPA \\
\hline $\mathrm{n}$ & 235 & 39 & 76 & 56 & 155 & 155 \\
\hline Mínimo & 32,20 & 46,10 & 44,50 & 36,60 & 45,70 & 20,00 \\
\hline Máximo & 105,00 & 77,50 & 111,50 & 133,50 & 100,80 & 43,00 \\
\hline Média & 58,85 & 53,05 & 65,19 & 67,57 & 65,36 & 38,77 \\
\hline Mediana & 56,80 & 50,20 & 62,75 & 65,55 & 63,80 & 40,00 \\
\hline Erro Padrão & 0,74 & 1,12 & 1,60 & 2,53 & 0,54 & 0,35 \\
\hline Coeficiente de variação & 19,18 & 13,22 & 21,43 & 28,00 & 10,31 & 11,10 \\
\hline Quartil 1 & 52,00 & 47,40 & 55,08 & 55,00 & 62,60 & 38,00 \\
\hline Quartil 3 & 64,40 & 58,40 & 71,40 & 78,10 & 68,05 & 42,00 \\
\hline$D$ & 0,1128 & 0,2221 & 0,1231 & 0,1122 & 0,1898 & 0,2564 \\
\hline$P$ & $<0,0001$ & $<0,0001$ & $<0,0001$ & 0,1124 & $<0,0001$ & $<0,0001$ \\
\hline
\end{tabular}

Legenda: $D$ : estatística do teste de Kolmogorov-Smirnov/Lilliefors; $P$ : probabilidade

Fonte: Elaborada pelos autores com dados da pesquisa, 2013.

Na UTI Adulto, 70,21\% dos atendidos estão entre 11,78 e 17,18 horas de enfermagem por paciente; na UTI Pediátrica, 69,64\% estão entre 11,78 e 19,88 horas de enfermagem por paciente; na Neonatal II, $92,31 \%$ estão entre 9,08 e 14,48 horas de enfermagem por paciente; e na Neonatal III, 63,16\% estão entre 11,78 e 17,18 horas de enfermagem por paciente. Em todas as unidades os pacientes com elevado número de horas de enfermagem são raros, sendo que de todas as unidades $79,31 \%$ deles estão a partir de 12 horas; $14,53 \%$ acima de 18 ; e somente $1,48 \%$ estáo a partir de 24 horas de enfermagem (Gráfico 1).

O NAS médio foi dependente do turno somente para a UTI Neonatal II e III, sendo que em ambas a manhã apresentou os maiores valores de NAS.
Enquanto na UTI Neonatal II a manhã atingiu NAS médio de 55,19; a tarde e a noite atingiram, respectivamente, 52,34 e 50,85 , sem diferenças significativas entre os dois turnos. Já na UTI Neonatal III, a manhã também atingiu o maior NAS médio, de 70,42; enquanto a tarde e a noite chegaram a 60,92 e 63,62, respectivamente, sem diferenças entre os dois turnos. Para as demais UTI avaliadas não houve diferença entre os turnos. Na comparação entre as quatro UTI foram observadas diferenças no valor do NAS médio, com a UTI Neonatal III com 65,19 e a UTI Pediátrica com 67,57, apresentando a maior média e sem diferenças entre si, seguidas da UTI Adulto com 58,85 e da UTI Neonatal II com 53,05, que, individualmente, foram distintas das demais (Tabela 2 ). 
Gráfico 1: Frequência de pacientes em diferentes intervalos de tempo de trabalho de enfermagem em função do Nursing Activities Score (NAS) em unidades de terapia intensiva de um hospital universitário brasileiro em 2013.

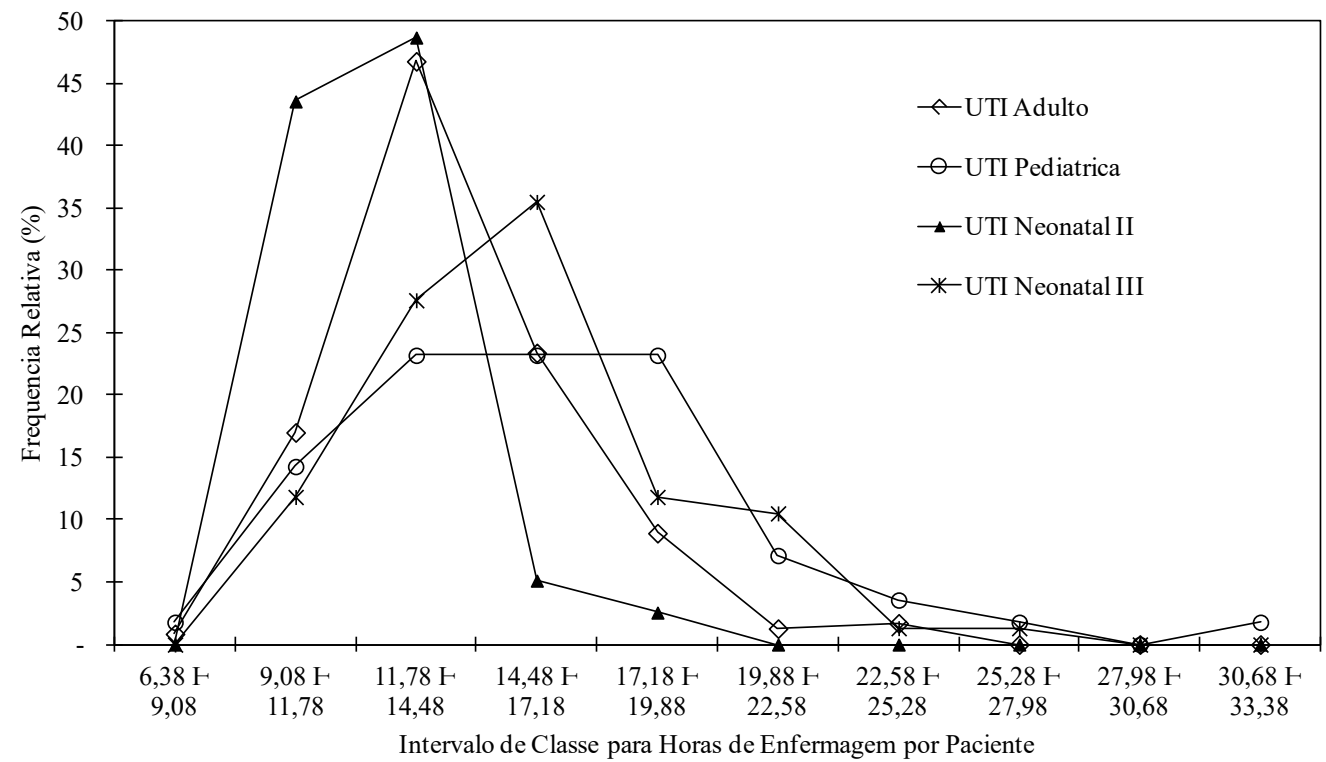

Fonte: Elaborado pelos autores com dados da pesquisa, 2013.

Tabela 2: Pontuação e dimensionamento para Unidades de Terapia Intensiva (UTI) de um hospital universitário brasileiro em 2013 em função do horário de classificação dos pacientes a partir do Nursing Activities Score (NAS).

\begin{tabular}{|c|c|c|c|}
\hline \multirow{2}{*}{ UTI } & \multirow{2}{*}{ Turno } & \multicolumn{2}{|c|}{ Média* } \\
\hline & & NAS (n) & Número de funcionários (n) \\
\hline \multirow{4}{*}{ Adulto } & Manhã & $57,34(89)$ & $89,41(3)$ \\
\hline & Tarde & $58,46(86)$ & $91,14(3)$ \\
\hline & Noite & $61,65(60)$ & $95,77(2)$ \\
\hline & Estatística (P) & $\chi^{2}=2,66(0,2600)$ & $\chi^{2}=0,69(0,7066)$ \\
\hline \multirow{4}{*}{ Neonatal Tipo II } & Manhã & $55,19(15) \mathrm{a}$ & $14,35(3)$ \\
\hline & Tarde & $52,34(14) \mathrm{b}$ & $13,70(3)$ \\
\hline & Noite & $50,85(10) b$ & $13,22(2)$ \\
\hline & Estatística (P) & $\chi^{2}=6,36(0,0415)$ & $\chi^{2}=1,44(0,4857)$ \\
\hline \multirow{4}{*}{ Neonatal Tipo III } & Manhã & $70,42(290) \mathrm{a}$ & $36,70(3)$ \\
\hline & Tarde & $60,92(29) \mathrm{b}$ & $33,38(3)$ \\
\hline & Noite & $63,62(18) b$ & $33,09(2)$ \\
\hline & Estatística $(P)$ & $\chi^{2}=8,43(0,0147)$ & $\chi^{2}=3,17(0,2043)$ \\
\hline \multirow{5}{*}{ Pediátrica } & Manhã & $64,56(20)$ & $27,02(3)$ \\
\hline & Tarde & $66,26(23)$ & $27,72(3)$ \\
\hline & Noite & $74,53(13)$ & $30,90(2)$ \\
\hline & Estatística (P) & $\chi^{2}=1,89(0,3894)$ & $\chi^{2}=2,03(0,3628)$ \\
\hline & $\mathrm{n}$ & Média geral dos turnos & funcionário.leito ${ }^{-1}$ (n) \\
\hline Adulto & 235 & $58,85 \mathrm{~b}$ & $3,06(3) b$ \\
\hline Neonatal II & 39 & $53,05 \mathrm{c}$ & $2,76(3) b$ \\
\hline Neonatal III & 76 & $65,19 \mathrm{a}$ & $3,46(2) \mathrm{a}$ \\
\hline \multirow[t]{2}{*}{ Pediátrica } & 56 & $67,57 \mathrm{a}$ & $3,53(2) \mathrm{a}$ \\
\hline & Estatística $(\mathrm{P})$ & $\chi^{2}=36,21(<0,0001)$ & $\chi^{2}=18,62(0,0003)$ \\
\hline
\end{tabular}

Legenda: $P$ : probabilidade, $\chi^{2}=$ Qui-quadrado para o teste de Kruskal-Wallis.

*Valores médios seguidos de letras distintas na coluna para cada comparaçáo são diferentes baseados no teste de Dunn $(P<0,05)$.

Fonte: Elaborada pelos autores com dados da pesquisa, 2013. 
Não houve diferença no dimensionamento entre os turnos de trabalho de cada uma das UTI (Tabela 2). Quando a comparação foi realizada na base de funcionários/mês/dia, observamos diferenças entre as UTI, com a Pediátrica $(3,53)$ e a Neonatal III $(3,46)$ apresentando a maior carga de trabalho e iguais entre si; e as UTI Adulto $(3,06)$ e Neonatal II $(2,76)$, as menores cargas e iguais entre si, e diferentes das demais (Tabela 2).

\section{Relação entre NAS e SCP adulto}

Os dados do SCP mostraram uma amplitude de 20 a 43 , com média de 38,77 pontos, e distribuição não normal o que representou uma variação de 5,6 a 17,90 horas de carga de trabalho, com média de 15,91 horas por paciente. O NAS mostrou uma amplitude de 45,7 a 100,8 pontos, com média de 65,36 , e distribuição não normal - representando uma variaçáo na carga de trabalho de 10,96 a 24,19 horas por paciente e média de 15,69 horas por paciente.

Já o número de horas por paciente diferiu entre os dois modos de classificação, com mediana de 17,9 para o SCP e de 15,31 para o NAS ( $U=2057, \mathrm{P}=0,0095$, Tabela 3 ); sendo que o número de horas entre os dois sistemas foi correlacionado entre si, mas de magnitude moderada ( $r s=0,4553 ; P<0,0001)$, como também ocorreu para os escores dos dois sistemas $(r s=0,4856 ; P<0,0001)$.

Tabela 3: Comparação entre as pontuações obtidas com o Sistema de Classificação de Pacientes Adultos de Santos (SCP) e com o Nursing Activities Score (NAS) e os números de funcionários obtidos no dimensionamento de pessoal em uma unidade de terapia intensiva de adultos.

\begin{tabular}{|c|c|c|c|c|c|c|c|}
\hline \multirow[t]{2}{*}{ Dia (n) } & \multirow[t]{2}{*}{ Score Médio NAS } & \multicolumn{4}{|c|}{ Frequência relativa (\%) dos tipos de cuidado do SCP } & \multicolumn{2}{|c|}{$\begin{array}{l}\text { Dimensionamento } \\
\text { (funcionário) }\end{array}$} \\
\hline & & Intensivo & Semi-intensivo & Intermediário & Mínimo & NAS & SCP \\
\hline $1(26)$ & 69,92 & 61,54 & 38,46 & 0,00 & 0,00 & 109,07 & 110,95 \\
\hline $2(27)$ & 68,34 & 74,07 & 25,93 & 0,00 & 0,00 & 106,61 & 119,01 \\
\hline $3(26)$ & 64,16 & 69,23 & 26,92 & 3,85 & 0,00 & 100,09 & 114,79 \\
\hline $4(25)$ & 62,80 & 56,00 & 40,00 & 4,00 & 0,00 & 97,96 & 106,21 \\
\hline $5(24)$ & 63,70 & 62,50 & 29,17 & 4,17 & 4,17 & 99,37 & 108,59 \\
\hline $6(27)$ & 63,02 & 59,26 & 29,63 & 11,11 & 0,00 & 98,31 & 106,27 \\
\hline Média & 65,32 & 63,77 & 31,68 & 3,85 & 0,69 & $101,90^{* *}$ & $110,98^{* \star}$ \\
\hline Mediana & 63,93 & 62,02 & 29,40 & 3,92 & 0,00 & 99,73 & 109,78 \\
\hline
\end{tabular}

** As médias foram diferentes baseadas no teste $t$ de Student para dados pareados $(t=5,05, P=0,0039)$.

Fonte: Elaborada pelos autores com dados da pesquisa, 2013.

Já a comparação entre o NAS e o SCP, para os seis dias, mostrou que o SCP gera um dimensionamento para os 30 leitos, maior que o NAS $(t=3,2009$, $P=0,0094$, Tabela 3); sendo que os dados de dimensionamento de cada dia obtidos não foram correlacionados entre si $(r=0,8285 ; P=0,0583)$. $\mathrm{O}$ modelo de regressão mostrou-se significativo, e que cada ponto do SCP equivale a 1,616 pontos do NAS (Gráfico 2).

Observa-se que nos 6 dias de coleta, os pacientes classificados como cuidados intensivos tiveram maior frequência comparados aos demais tipos de cuidado (Tabela 3). Em apenas um dia houve a classificação de um paciente em cuidados mínimos. No geral, a maioria dos pacientes é classificada como cuidado intensivo (média $=63,77 \%$ ) ou semi-intensivo (média=31,68\%). Não houve dependência entre o dia de avaliação e a frequência de pacientes em cada uma das classes de cuidado $(G=8,60 ;$ g.l. $=15 ; P=0,8970)$, mostrando a repetição do padrão entre os dias.
O NAS médio de cada tipo de cuidado foi de 10,97 para cuidados mínimos $(\mathrm{n}=1) ; 12,77$ para cuidados intermediários; 15,23 para cuidados semi-intensivos; e de 16,14 para cuidados intensivos. Exceto para os cuidados intensivos, o NAS estimou uma carga de trabalho de enfermagem maior do que aquela preconizada.

\section{Discussão}

De modo geral, não foram encontradas diferenças nas pontuaçóes verificadas nos diferentes horários de avaliação realizados nas UTI, exceto na UTI Neonatal em que as pontuaçóes referentes ao período da manhã foram estatisticamente diferentes dos outros períodos. Esse resultado na UTI Neonatal não era esperado diante do caráter retrospectivo do NAS para as últimas 24 horas $^{1}$, que, a princípio, deveria ter resultados iguais ou 
próximos em todos os turnos. Um dos fatores que pode ter afetado esse resultado é o baixo número de dias avaliados, pois se avaliados mais dias, eventualmente dias de maior dependência poderiam ser diluídos na amostra.

Gráfico 2: Relação entre o Sistema de Classificação de Pacientes Adultos de Santos (SCP) e o Nursing Activities Score (NAS) em uma unidade de terapia intensiva adulta de um hospital universitário brasileiro em 2013.

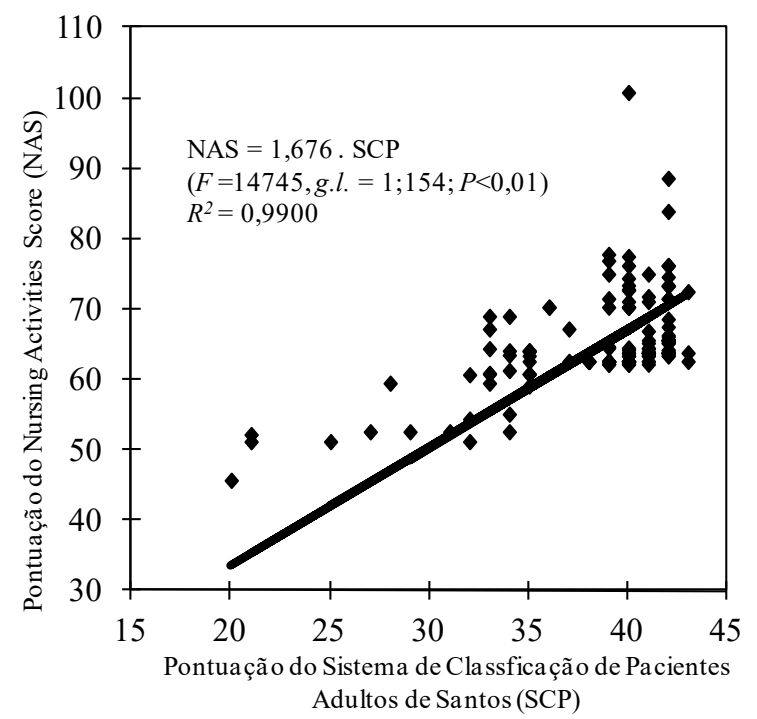

Fonte: Elaborado pelos autores com dados da pesquisa, 2013.

Outro fator é a melhor capacidade de classificação dos enfermeiros do período da manhã comparada com aqueles de outros turnos. O perfil dos pacientes em UTI é bem típico e provavelmente teria pouco efeito sobre os escores médios, embora não tenhamos caracterizado os pacientes nesse período, o que prejudica a discussão. Esse resultado provavelmente está mais relacionado à capacidade e ao maior conhecimento do histórico do paciente pela equipe da neonatologia no período da manhã. Quando Debergh et al. ${ }^{18}$ avaliaram o NAS em unidades pediátricas e médicas para diferentes períodos de trabalho também encontraram diferenças entre turnos, com a manhá apresentando os maiores valores médios, embora no estudo o instrumento tenha sido adaptado para a verificação da carga de trabalho por turno e não por dia, como realizado por nós. Estudos mais amplos ainda são necessários para compreender essas oscilações.

O NAS tem sido pouco utilizado em UTI de neonatologia. Os valores obtidos na UTI Neonatal III estáo próximos aos da literatura, ao contrário dos da UTI Neonatal II, que estão abaixo - fato que ocorre devido à baixa complexidade desta unidade. Há poucos registros para neonatologia no Brasil, destacando-se, para UTI neonatologia, um escore médio de 86,78 - em unidades de internação de neonatologia com diferentes complexidades há registro de escore médio variando entre 29,07 em unidades de baixo risco, 32,52 para as de médio risco, 41,52 para as de alto risco e 85,74 para as unidades de isolamento ${ }^{10}$, reforçando as diferenças do NAS entre unidades de complexidade diferencial, como o encontrado por nós. O mesmo estudo também demonstrou uma defasagem do pessoal de enfermagem, embora não tenhamos dimensionado o setor por particularidades do serviço, como rodízio de servidores entre as unidades de Berçário e neonatologia. Antunes et al. ${ }^{11}$ dimensionaram todo o serviço de neonatologia do Hospital Universitário e observaram uma necessidade de acréscimo de $79 \%$ de pessoal. Fugulin et al. ${ }^{19}$ encontraram um escore médio de 68,9 em outra instituição cuja necessidade de aumento de custos com pessoal era de 30\%. Instrumentos como o NAS ainda são pouco utilizados na neonatologia, e na literatura como um todo - realidade também presente em outros instrumentos de mensuração de carga de trabalho em neonatologia ${ }^{9,20,21}$.

Como em neonatologia, o uso do NAS em pediatria ainda é escasso e o único trabalho realizado no Brasil encontrou valores médios de 56,00 e 58,8021. Para ICU Pediátrica na Bélgica foi registrado um NAS médio de $57^{18}$, demonstrando que os valores encontrados por nós estão bem acima do perfil da última instituição.

Quanto às UTI Adulto, os valores encontrados por nós estáo dentro dos estudos brasileiros, nos quais a pontuação varia de $45,4^{22}$ a $104,1^{23}$, sendo que diversos fatores têm afetado o resultado do NAS, como a presença de sepse, o tipo de hospitalização, o tempo de internação, os desfechos de alta da UTI ou de óbito, o sistema público versus o privado de admissão ${ }^{24,25,26,27}$.

A diferença no NAS, em função do turno na neonatologia, pode somente ser um reflexo do perfil das equipes ou da organização do próprio serviço. No período da manhã todas as condutas são revistas e o histórico do paciente acaba por ser mais conhecido por cada membro da equipe. Uma vez que esse foi o primeiro contato das equipes com o instrumento - o qual fora apresentado e discutido anteriormente para a realização do dimensionamento ${ }^{15}$-, a própria inexperiência dos integrantes pode ser fator relevante no processo.

Apesar da grande aplicabilidade do NAS, Palese et al. ${ }^{28}$ observaram que ele pode não ser aplicável ou pode demandar alteraçóes dependendo do local a ser aplicado, como na populaçáo avaliada por eles. Fatores como o tempo de aplicação têm sido relacionados a dificuldades na aplicação de sistemas de classificação de pacien$\operatorname{tes}^{29}$, o que pode refletir uma necessidade de reavaliação do instrumento e sua confiabilidade na população brasileira ou em grupos distintos de ICU; ou ainda a 
necessidade de um processo de educação continuada mais intenso antes do seu uso.

A comparação entre o SCP e o NAS na UTI Adulto mostrou a forte relação entre os sistemas. Fugulin et al. ${ }^{30}$ realizaram uma validação das cargas preconizadas pelo Cofen para dimensionamento utilizando o SCP, com observação de quadro menor de pessoal que o recomendado na maioria das unidades avaliadas, mas adequação do quadro preconizado pelo Cofen. O perfil encontrado, com baixa frequência de cuidados mínimos e intermediários pelo SCP, é condizente com outros estu$\operatorname{dos}^{31,32}$. Cada ponto do SCP equivaleu a 1,676 do NAS, o que corresponde 0,4022 horas de carga de trabalho; considerando que cada ponto do NAS equivale a 14,4 minutos, e do SCP a 24,13 minutos; embora a baixa representatividade de cuidados mínimos e cuidados intermediários dificulte a generalização. Cerca de 76\% dos pacientes de cuidados intensivos tiveram a carga de trabalho menor estimada pelo NAS.

Os dois métodos de dimensionamento mostraram resultados diferentes entre si e não encontramos literatura que permita discutir o efeito do uso dos dois instrumentos. Apesar disso, quando Yanaba et al..$^{32}$ utilizaram o SCP para dimensionamento de uma UTI, observaram um excesso de profissionais, diferente do observado na unidade avaliada aqui. Ainda são necessários estudos comparativos entre instrumentos para a realidade brasileira. Mas, aparentemente, o NAS leva a uma estimativa maior de carga horária, embora não seja possível, a partir dos nossos dados, estipular o método mais adequado.

\section{Conclusão}

Os resultados mostram que não ocorreram diferenças significativas entre a carga de trabalho de enfermagem entre os turnos de trabalho nas UTI avaliadas; exceto na neonatologia, onde se observou uma maior carga de trabalho no turno da manhã. O NAS deveria ser pouco afetado pelo turno de aplicação, principalmente pelo caráter retroativo. Estudos adicionais são necessários para confirmar essa tendência na neonatologia.

Tal efeito pode ser mais marcado em dimensionamentos de urgência ou que utilizem um banco de dados restrito como o nosso, onde o perfil do paciente pode ser um fator importante. Tanto o NAS como o SCP, na UTI Adulto, foram de fácil aplicação e têm um papel importante na determinação da carga de trabalho e no dimensionamento de pessoal nessas unidades. Apesar disso, o SCP levou a um maior dimensionamento, talvez pela sua restrição de classificação em poucas faixas de cuidado e por consequência de carga de trabalho; já que para pacientes do tipo intensivo, o SCP tende a superestimar a carga de trabalho quando comparado ao NAS. Apesar da ausência de diferenças no dimensionamento, os instrumentos podem gerar custos financeiros relevantes para as instituiçóes, e devem ser mais bem compreendidos para o uso em ICU.

\section{Agradecimentos}

Agradecemos às equipes de enfermagem das unidades de terapia intensiva e à Diretoria de Enfermagem do Hospital de Clínicas de Uberlândia pela colaboração no estudo e liberação dos dados. Aos revisores pelas sugestóes.

\section{Referências}

1. Miranda DR, Nap R, Rijk A, Schaufeli W, Iapichino G. Nursing Activities Score (NAS). Crit Care Med. 2003;31(2):374-82.

2. Santos F, Rogenski NMB, Baptista CMC, Fugulin FMT. Sistema de classificaçáo de pacientes: proposta de complementação do Instrumento de Fugulin et al. Rev Lat Am Enfermagem. 2007;15(5):980-5.

3. Queijo AF, Padilha KG. Nursing Activities Score (NAS): adaptação transcultural e validação para a língua portuguesa. Rev Esc Enferm USP. 2009;43:1018-25.

4. Reich R, Vieira DF, Lima LB, Rabelo-Silva ER. Nursing workload in a coronary unit according to the Nursing Activities Score. Rev Gaúcha Enferm. 2015;36(3):28-35.

5. Brito AP, Guirardello EB. Nursing workload in an inpatient unit. Rev Lat Am Enfermagem. 2011;19(5):1139-45.

6. Lima MKF, Tsukamoto R, Fugulin FMT. Aplicação do nursing activities score em pacientes de alta dependência de enfermagem. Texto ContextoEnferm. 2008;17(4):638-46.

7. Daud-Gallotti RM, Costa SF, Guimarães T, Padilha KG, Inoue EN, Vasconcelos TN, et al. Nursing workload as a risk factor for healthcare associated infections in ICU: a prospective study. PLoS One. 2012;7(12):e52342.

8. Dini AP, Guirardello EB. Pediatric patient classification system: improvement of an instrument. Rev Esc Enferm USP. 2014;48(5):787-93.

9. Spence K, Tarnow-Mordi W, Duncan G, Jayasuryia N, Elliott J, King J, et al. Measuring nursing workload in neonatal intensive care. J Nurs Manag. 2006;14(3):227-34.

10. Nunes BK, Toma E. Assessment of a neonatal unit nursing staff: application of the Nursing Activities Score. Rev Lat Am Enfermagem. 2013;21(1):348-55.

11. Antunes AV, Mendes-Rodrigues C, Gomes FA, Rezende GJ, Braga IA, Teixeira LMR, et al. Dimensionamento de pessoal de enfermagem [relatório técnico]. Uberlândia: Universidade Federal de Uberlândia - Hospital de Clínicas de Uberlândia. 2013. 93 p. 
12. Brasil. Resolução da Diretoria Colegiada no 7 , de 24 de fevereiro de 2010. Dispóe sobre os requisitos mínimos para funcionamento de Unidades de Terapia Intensiva e dá outras providências [Internet]. Brasília, DF; 2010 [citado em 2016 jan 11]. Disponível em: https://goo.gl/qPaZTB

13. Brasil. Portaria MS/GM no 3.432 de 12 de agosto de 1998. Estabelece critérios de classificação para as Unidades de Tratamento Intensivo - UTI [Internet]. Diário Oficial da União. Brasília DF; 13 ago 1998 [citado em 2016 jan 11]:109-10. Disponível em: https://goo.gl/6m9ZGz

14. Cofen. Resolução Cofen-293/2004. Fixa e estabelece parâmetros para o dimensionamento do quadro de profissionais de enfermagem nas unidades assistenciais das instituiçóes de saúde e assemelhados [Internet]. Rio de Janeiro; 21 set 2004 [citado em 2016 jun 27]. Disponível em: https://goo. $\mathrm{gl} / \mathrm{UBHq} 5 \mathrm{~V}$

15. Antunes AV, Costa MN. Dimensionamento de pessoal de enfermagem em um hospital universitário. Rev Lat Am Enfermagem. 2003;11(6):832-9.

16. Gaidzinski RR. Dimensionamento de pessoal de enfermagem. In: Kurcgant P, organizadora. Administração em Enfermagem. São Paulo: E.P.U; 1991. p. 91-6.

17. Ayres M, Ayres M Jr, Ayres DL, Santos AAS. BioEstat: aplicaçóes estatísticas nas áreas das ciências biomédicas. Belém: ONG Mamirauá; 2007.

18. Debergh DP, Myny D, Van Herzeele I, Van Maele G, Reis Miranda D, Colardyn F. Measuring the nursing workload per shift in the ICU. Intensive Care Med. 2012;38(9):1438-44.

19. Fugulin FMT, Lima AFC, Castilho V, Bochembuzio L, Costa JA, Castro L, et al. Custo da adequação quantitativa de profissionais de enfermagem em Unidade Neonatal. Rev Esc Enferm USP. 2011;45:1582-8.

20. Bochembuzio L, Gaidzinski RR. Instrumento para classificação de recém-nascidos de acordo com o grau de dependência de cuidados de enfermagem. Acta Paul Enferm. 2005;18(4):382-9.

21. Campagner AOM, Garcia PCR, Piva JP. Use of scores to calculate the nursing workload in a pediatric intensive care unit. Rev Bras Ter Intensiva. 2014;26(1):36-43.

22. Beccaria LM, Contrin LM, Cesarino CB, Silva DC,
Silva APA, Werneck AL. Association between nursing workload and the patient prognosis in intense care unit. Bus Manag Rev. 2015;4(5):731-8.

23. Inoue KC, Kuroda CM, Matsuda LM. Nursing Activities Score (NAS): carga de trabalho de enfermagem em UTI e fatores associados. Cienc Cuid Saude. 2011;10(1):134-40.

24. Nogueira LS, Koike KM, Sardinha DS, Padilha KG, Sousa RMC. Carga de trabalho de enfermagem em unidades de terapia intensiva públicas e privadas. Rev Bras Ter Intensiva. 2013;25(3):225-32

25. Padilha KG, Sousa RM, Queijo AF, Mendes AM, Reis Miranda D. Nursing Activities Score in the intensive care unit: analysis of the related factors. Intensive Crit Care Nurs. 2008;24(3):197-204

26. Altafin JAM, Grion CMC, Tanita MT, Festti J, Cardoso LTQ, Veiga CFF, et al. Nursing Activities Score and workload in the intensive care unit of a university hospital. Rev Bras Ter Intensiva. 2014;26(3):292-8.

27. Altafin JAM, Grion CMC, Kauss IAM, Anami EHT, Cardoso LTQ, Chiqueti F, et al. Increased nursing workload in patients with sepsis: A cohort study. Clinical Nursing Studies. 2016;4(1):33-9.

28. Palese A, Comisso I, Burra M, DiTaranto PP, Peressoni L, Mattiussi E, et al. Nursing Activity Score for estimating nursing care need in intensive care units: findings from a face and content validity study. J Nurs Manag. 2016;24(4):549-59.

29. Bosco CS, Toma E, Oliveira SMJV, Belli MAJ. Reliability of an instrument to classify newborns according to care complexity. Rev Esc Enferm USP. 2013;47(4):788-93.

30. Fugulin FMT, Rossetti AC, Ricardo CM, Possari JF, Mello MC, Gaidzinski RR. Tempo de assistência de enfermagem em Unidade de Terapia Intensiva: avaliação dos parâmetros propostos pela Resoluçáo COFEN no 293/04. Rev Lat Am. Enfermagem. 2012;20(2):325-32.

31. Perroca MG, Gaidzinski RR. Avaliando a confiabilidade interavaliadores de um instrumento para classificação de pacientes: coeficiente Kappa. Rev Esc Enferm USP. 2003;37(1):72-80.

32. Yanaba DS, Giúdice CAR, Casarin SNA. Dimensionamento da equipe de enfermagem em unidade de terapia intensiva para adultos. J Health Sci Inst. 2013;31(3):279-85

\section{Como citar este artigo:}

Mendes-Rodrigues C, Costa KES, Antunes AV, Gomes FA, Rezende G Jr, Silva DV. Carga de trabalho e dimensionamento de pessoal de enfermagem em unidades de terapia intensiva. Rev. Aten. Saúde. 2017;15(53):5-13. 